\title{
Late haemorrhage from the subclavian vein due to a fracture of the first rib
}

\author{
F. W. Rozendaal, H. J. Bonjer and H. A. Bruining ${ }^{1}$ \\ Department of Anaesthesiology and 'Department of General Surgery, University Hospital Dijkzigt, Rotterdam,
} The Netherlands

Injury, 1995, Vol. 26, 57-58, January

\section{Introduction}

Lesions of the subclavian vessels due to closed chest injury are rare. Berga et $\mathrm{al}^{1}{ }^{ }$, reported during a five year period a 0.2 per cent incidence of lesions of the subclavian artery, in patients with closed chest injury. Pain and swelling were consistent findings in patients with traumatic lesions of the subclavian artery. Absence of peripheral pulsations or murmurs were occasionally found. In spite of severe damage of the subclavian arter $y^{2}$, peripheral pulsations can be present because of extensive collateral circulation of the upper extremity. Fractures of the clavicle, first rib or second rib, were found in all patients with lesions of the subclavian artery. A correlation between either the localisation of the rib fracture, or the degree of dislocation of the fragments and the occurrence of a lesion of the subclavian artery, has not been found ${ }^{3}$.

\section{Case report}

A 45-year-old male driver of a car was admitted to hospital because he had been involved in a head-on collision. On admission the Glasgow Coma Score was 15, the blood pressure was $110 / 40 \mathrm{mmHg}$ and the pulse rate 110 beats per minute. The arterial oxygenation was 96 per cent. Radiological examination of the chest showed fractures of the second to ninth left ribs, a fracture of the midportion of the left clavicle and a fracture of the neck of the left scapula. There was a possible fracture of the left first rib. The chest radiograph showed a bilateral haemopneumothorax with extensive subcutaneous emphysema (Figure 1). The patient was intubated and ventilated mechanically. Bilateral thoracic drains were inserted. An emergency laparotomy was done because of haemodynamic instability; there was a ruptured spleen necessitating splenectomy. A tracheostomy was performed. Post-operatively the pulmonary condition of the patient deteriorated due to extensive lung contusion which required positive end expiratory pressure ventilation.

On the second day post-operatively, the left arm was warm and oedematous. The arterial circulation of the left arm was unaffected. Because venous thrombosis of the left axillary vein was suspected, the left arm was elevated.

On the fifth day post-operatively, the patient suddenly lost $2500 \mathrm{ml}$ of fresh blood through his left thoracic drain. An emergency left thoracotomy via the fifth intercostal space revealed a tear of the left subclavian vein caused by a fracture of the first rib.

The rupture of the subclavian vein was repaired primarily with Polypropylene $^{\mathbb{R}} 5.0$ suture. In addition, pleural flaps were sutured on the subclavian vein to protect the vessel against another lesion from the fragments of the first rib.

Inspection of the subclavian artery, arcus aortae and aorta descendens showed no other damage. The post-operative course was uneventful, and the patient was discharged from the hospital 1 month after the accident. There were no signs of venous thrombosis, arterial insufficiency or neurological damage.

\section{Discussion}

Isolated lesions of the subclavian vein in patients with closed chest trauma have never been reported to our knowledge. In our patient, the massive bleeding of the subclavian vein was life-threatening and could be diagnosed early because the thoracic drain was still in situ. The late rupture of the subclavian vein was probably a consequence of the movement of the fracture fragments of the first rib or clavicle, or both, causing either a new lesion

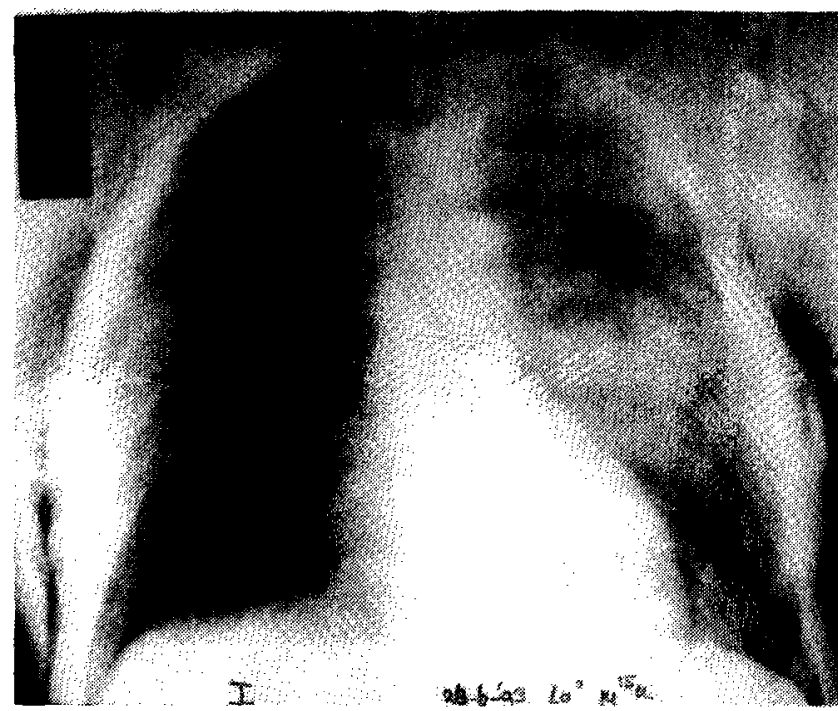

Figure 1. Radiograph showing fracture of clavicle, scapulae. second to ninth ribs, possible first rib fracture (left hemithorax) bilateral lung contusions and subcutaneous emphysema. 
of the subclavian vein, or an erosion of a thrombus sealing off a previous rupture of the subclavian vein. After the repair of the subclavian vein it is recommended that the vein is protected with pleural flaps to prevent injury.

In conclusion. ruptures of the subclavian vein in patients with closed chest injury can cause life-threatening bleeding up to several days after the injury.

\section{References}

1 Bega C, Prat S, Ninot S et al. The arterial complications of closed injuries to the shoulder girdle. Angiologica 1992; 44: 139.
2 Buscaglia LC, Walsh JC, Wilson J. D. et al. Surgical management of subclavian artery injury. Am J Surg 1987; 154: 88.

3 Livoni JP and Barcia TC. Fracture of the first and second rib; incidence of vascular injury relative to the type of fracture. Radiology 1982; 145: 31.

Paper accepted 14 July 1994.

Requests for reprints should be addressed to: F. W. Rozendaal, Department of Anaesthesiology, University Hospital Dijkzigt, Dr. Molewaterplein 40, 3015 GD Rotterdam, The Netherlands. 